\title{
Multiagent System Architecture for Short-term Operation of Integrated Microgrids
}

\author{
Mohammad Ali Fotouhi Ghazvini*. Reza Abedini* \\ Tiago Pinto*. Zita Vale* \\ * GECAD - Knowledge Engineering and Decision-Support Research Center \\ IPP - Polytechnic Institute of Porto, Porto, Portugal; e-mail: \{arach, mafgh, tmcfp, zav\}@isep.ipp.pt).
}

\begin{abstract}
The forthcoming smart grids are comprised of integrated microgrids operating in grid-connected and isolated mode with local generation, storage and demand response (DR) programs. The proposed model is based on three successive complementary steps for power transaction in the market environment. The first step is characterized as a microgrid's internal market; the second concerns negotiations between distinct interconnected microgrids; and finally, the third refers to the actual electricity market. The proposed approach is modeled and tested using a MAS framework directed to the study of the smart grids environment, including the simulation of electricity markets. This is achieved through the integration of the proposed approach with the MASGriP (Multi-Agent Smart Grid Platform) system.
\end{abstract}

Keywords: Day-ahead market, Electricity market, Microgrids, Multiagent systems, Smart grids.

\section{NOMENCLATURE}

b Buses

$i \quad$ Microgrids/Microgrid central controllers (MGCC).

j Virtual power players (VPP).

$q \quad$ Distributed generation (DG) units.

$s \quad$ Energy storage systems (ESS).

$k \quad$ Load aggregators (LA).

$R \quad$ Regulated retail electricity prices offered by the VPPs to LAs (€/MWh).

$d_{b} \quad$ Electricity purchase at bus $b(\mathrm{MWh})$.

$p_{l / g} \quad$ Load/generation power (MW).

$\Omega_{j} \quad$ Set of the buses that the VPP $j$ is serving loads or managing DGs and ESSs in them.

$\Omega_{j}^{q / s} \quad$ Set of DGs/ESSs owned by VPP $j$.

$\Omega_{i} \quad$ Set of the buses located within the electrical boundary of microgrid $i$.

\section{INTRODUCTION}

Microgrids are distributed low voltage power networks that mainly rely upon internal DG units and ESSs for supplying their loads. They are electrically connected to the power grid at the distribution level and have clear electrical boundaries.
The basic functions and key security requirements of a power system should be maintained during short-term operation of a microgrid, whether the microgrid is interconnected to the power grid or operates as an isolated system. The power grid sees the microgrid as a single controllable entity behaving as aggregated loads or sources (Logenthiran, 2012). Lower net operating cost encourages the MGCC to cooperate with other interconnected microgrids (Dimeas and Hatziargyriou, 2005). Establishing an effective coordination mechanism between microgrids and the main distribution system is a critical challenge in the short-term operation of integrated microgrids.

This paper aims at analyzing the interaction between the local entities of a microgrid and the coordination among the integrated microgrids before entering the external day-ahead market. In order to commercialize the implementation of microgrids, we need the models that clearly define and characterize the behavior of participants in the market environment, where the entities usually seek their short-term and long-term financial benefits.

MAS is a distributed computational intelligent approach suitable for modeling the operation of microgrids in shortterm markets (Logenthiran and Srinivasan, 2012, Logenthiran, 2012). It represents loosely connected network of interacting distributed intelligent hardware and software agents (Logenthiran, 2012, Wooldridge, 2008). The smart grid concept is also easily implemented in this type of platform, due to its ability in modeling the autonomous decision making entities. The MAS is used in this paper to model the short-term operation of integrated microgrids in a multi-market environment.

The model in (Dimeas and Hatziargyriou, 2005) introduces agent-based approaches as alternatives to centralized control 
and management of microgrids. The practical implementation of integrated microgrids has been tested with a laboratorybased case study in (Bo et al., 2012), assessing the efficiency of the control and protection strategies in relation to the DG and ESS technologies. The short-term operation of a microgrid containing DGs and ESSs is modeled in (Fotouhi Ghazvini et al., 2012), and it has been noted that the ESSs are more profitable when the MGCC plans to increase the share of intermittent units. Microgrids attain more benefits from resource sharing among electrically interconnected microgrids (Logenthiran and Srinivasan, 2012). A hierarchical control scheme is used in (Logenthiran and Srinivasan, 2012, Logenthiran, 2012) for optimal scheduling of DGs and the power exchange between integrated microgrids and the power grid. An agent-based market clearing model for DR exchange is proposed in (Duy Thanh et al., 2012).

The operation of integrated microgrids in the market environment is modeled with a MAS approach. The proposed approach considers three different levels of negotiation, which include the internal operation of each independent microgrid, by performing the internal dispatch. The other two levels consider external negotiations with entities located outside the microgrid, namely nearby microgrids, and the electricity market players. The model is based on the advances of smart grids and considers microgrids containing ESSs and the DR programs offered by VPPs.

The proposed approach is integrated with MASGriP (Oliveira et al., 2012), a smart grid environment simulator, which provides an interface with the MASCEM (Multi-Agent Simulator for Competitive Electricity Markets) (Vale et al., 2011b), a system that models the electricity markets, using real data in order to test and validate the proposed approach.

\section{MICROGRID OPERATION}

Microgrids ease and promote the integration of renewable energy units, such as photovoltaic, wind and fuel cell generations into electricity grids. The main function of a microgrid is to ensure stable operation during faults and various network disturbances and the main challenge is managing the volatility of energy generation. The ESSs are implemented to facilitate the large integration of intermittent DGs, such as wind and solar systems.

Microgrids are usually connected to the power delivery system at a point of common coupling (PCC), thus appearing as a controllable single subsystem to the utility grid (Salam et al., 2008). In many power systems, interconnected microgrids disconnect from the grid while the system is under the stress of an abnormal condition. They operate in an island mode to avoid power supply interruption and eliminate possible voltage digs. DGs enhance the reliability of the system by providing backup generation for a microgrid during this operation mode. The small size of microgrids makes the operation more challenging, with physical implications for the performance of the power system. Load changes are large relatively to the total load, making frequency control more challenging (Bollen et al., 2009).

\subsection{Microgrid central controller}

The MGCCs are at the top level of microgrids' control and management structure and coordinates the operation between the DGs, ESSs, loads and the utility grid. It has to optimize the operation of the microgrid, while maintaining the acceptable voltage and frequency levels under both gridconnected and islanded mode. The boundary of a microgrid is determined by the set of buses that are under the management of a MGCC. Making decisions for the islanding operation based on economic or technical issues is another functionality of the MGCCs (Rasheduzzaman et al., 2012).

\subsection{Virtual power players}

The VPP approach supports the operation of microgrids with various LAs, entities serving the end-use customers, DGs and ESSs, which are the main elements of the generation side. These entities operate under the management and supervision of VPPs (Oliveira et al., 2012). In the proposed model, the responsible administrative entity for demand side management is the VPP. It performs the DR programs by offering financial incentives to the LAs to shift the loads to lower price periods. The main functionality assumed for the VPPs is the determination of the amount of financial incentives for demand reduction of LAs.

LAs aggregate the demand of end-users and have the flexibility to accept DR commands from the VPPs. It is assumed that a VPP can manage several LAs, DGs and ESSs, or it can be net consumer/producer (buyer/seller). The LAs in this model are shielded from the market price changes and the financial risks arise.

Equation (1) shows the objective of the VPPs before entering the internal market of a microgrid. They maximize their expected profit by offering financial incentive-based DR programs to the LAs. The proposed load shifting DR program leads the optimal demand bids of the VPPs at the nodes of a microgrid. The $L M P_{b}^{t}$ in (1) is the VPPs' expected LMP at bus b.

The financial incentive function changes at each bus at different time periods. It depends on the rival VPPs' offering and bidding strategies at the bus. The function FI(.) gives the financial incentive that the VPP offer for demand reduction; the coefficients of this function are the variables of the optimization problem. $\Delta l_{k}^{t}$ is the total demand reduction of LA $k$, but $D R_{b}^{t}$ is the demand reduction below the baseline consumption that receives financial incentives from the DR programs. $\mathrm{C}_{q}(\cdot)$ in (1) is the cost function of DG q. $p_{i n, s}^{t}$ is the energy bought and stored during hour $\mathrm{t}$ by VPP $j$ to supply loads in the future time periods.

$$
\begin{aligned}
& \text { Maximize } \sum_{t}\left[\sum_{k} R_{k}^{t}\left(l_{k}^{t}-\Delta l_{k}^{t}\right)-\sum_{b \in \Omega_{j}} L M P_{b}^{t} \cdot d_{b}^{t}\right. \\
& \left.-\sum_{q \in \Omega_{j}^{q}} \mathrm{C}_{q}\left(p_{q}^{t}\right)-\sum_{b \in \Omega_{j}} \mathrm{FI}_{b, t}\left(D R_{b}^{t}\right)-\sum_{s \in \Omega_{j}^{s}} p_{i n, s}^{t} \cdot L M P_{b}^{t}\right]
\end{aligned}
$$




\section{INTEGRATED MICROGRIDS}

The operation of integrated microgrids in the short-term electricity markets is modeled in three steps. The MGCC clears the internal market considering the offers and bids of the VPPs, their capabilities and restrictions and the network constraints. Prior to submitting the bids for the external dayahead market, the interconnection allows the negotiation among the integrated microgrids. The proposed framework can be used for intraday markets if the MGCCs can clear the internal market and negotiate with neighbour MGCCs before each phase of the intraday market. This condition is demanded only if the MGCCs wants to serve part of the loads from the grid.

\subsection{Microgrids' internal market}

In the internal market of each microgrid the MGCC clears the market while ensuring the security and reliability of the microgrid. This market is for the VPPs to submit their offers and bids with regard to the internal market particular rules and policies. The VPPs submit price-responsive demand bids at each bus for price elastic loads and inelastic demand bids for the loads that accept energy at any cost. The supply offer and demand bid curves at the buses with more than one serving VPPs are aggregated before the market clearing procedure. Within this procedure, the load dispatch for responsive loads and the generation dispatch for the controllable DGs will be calculated. The result of the market clearing procedure is the LMPs and the schedules for power generation and consumption of each VPP.

The objective of the MGCC for internal market clearing would be to minimize the net operating cost (2), subject to the power flow constraints (Liu et al., 2009). In (2), $\mathrm{C}_{b}(\cdot)$ is the aggregated generation cost function of the VPPs at each bus and $\mathrm{B}_{b}(\cdot)$ is the aggregated consumption benefit function of the VPPs at each bus. The expected cost and benefit function of the interconnected microgrids at the joint buses are also aggregated with these functions. The hourly LMP for the real power at each bus is the Lagrange multiplier associated with the real power balance constraints at that bus (Liu et al., 2009).

$$
\text { Minimize } \sum_{t}\left[\sum_{b \in \Omega_{i}} \mathrm{C}_{b}\left(p_{g, b}^{t}\right)-\sum_{b \in \Omega_{i}} \mathrm{~B}_{b}\left(p_{l, b}^{t}\right)\right]
$$

\subsection{Negotiation among $M G C C s$}

MGCCs of interconnected microgrids enter a negotiation procedure before finalizing the hourly offers and bids for the external day-ahead market. Resource sharing may help them operate more cost effectively. The MGCCs run the market clearing in the internal market based on the expected offers and bids of the neighbour interconnected microgrids. But, during the negotiation they may find cheaper energy or find out that the neighbor MGCC is ready to buy electricity at higher prices. During this procedure the MGCCs finalize their transactions for the day-ahead market.

Each MGCC performs an analysis of the market, to obtain the expected prices in the next sessions of the external day-ahead market. This value is used as reference for the analysis of the possible deals to be negotiated. If the proposals offered by the neighbour MGCCs are more favourable than the expected market prices, the MGCCs can choose to buy or sell some energy among them, obtaining better deals than they would have if negotiated the entire amount in the market. Besides this strategic analysis, players can use negotiation techniques when trying to obtain the best deals with their neighbours. For that we use several personality based strategies for agents' behaviour in the negotiations: (i) Determined - prices remain constant throughout the period of negotiation; (ii) Anxious - high changes to the price are made after a small amount of trading time; (iii) Moderated - small changes to the price are made in an intermediate stage of negotiation period; (iv) Gluttonous - the price is significantly changed, but only in late trading.

Players' price change tendencies using each of the considered negotiation strategies are presented in fig. 1. These strategies allow MGCCs to try different approaches when negotiation with their neighbours.

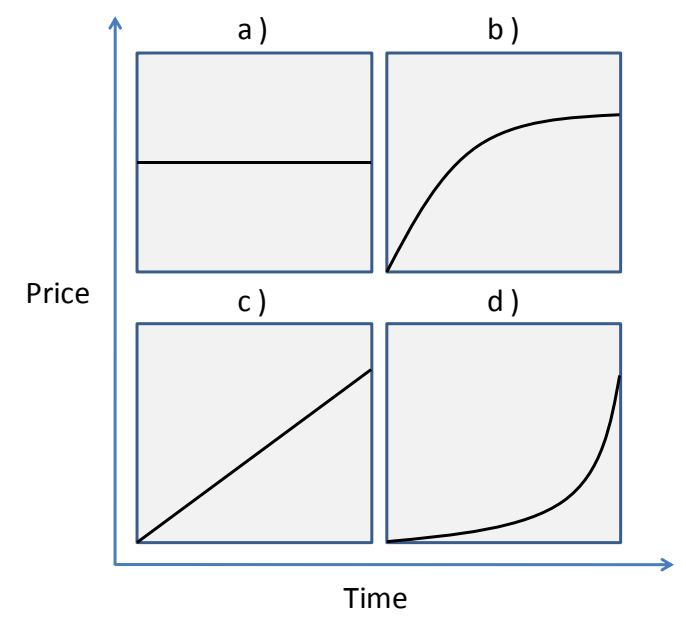

Fig. 1. Players' negotiation strategies tendencies. a) Determined, b) Anxious, c) Moderated, d) Gluttonous (Vale et al., 2011a)

The output of the two aforementioned steps is the hourly offers that each MGCC submits to the DNO at the connecting buses of the microgrids with the upstream network.

\subsection{External day-ahead market}

The DNO is responsible for the operation of low/medium voltage distribution networks same as the ISO at the transmission level (Logenthiran, 2012). The market operator can be a separate entity than the DNO. In our model it is assumed that the DNO is also carrying the responsibilities of a market operator.

In the pool-based external day-ahead market, the DNO receives the hourly offers from the MGCCs, concerning the expected production/consumption and corresponding price. (Conejo et al., 2010). The DNO uses a multi-period market clearing tool to produce a feasible dispatch, and maximizing the social welfare (Conejo et al., 2010). It determines the 
hourly prices based on the double-sided auction involving the supply offers and the demand bids of the interconnected MGCCs and the distribution system (Duy Thanh et al., 2012).

\section{PROPOSED MULTI-AGENT-BASED SCHEME}

The operation of power systems becomes complex with the smart grid technologies and requires more advanced control and management schemes, especially in a competitive market environment. MAS is an appropriate platform for implementing the smart grid concepts, because it provides intelligent decision making and adaptive local control (Oliveira et al., 2012).

The proposed model represents the relation between interconnected microgrids. The intelligent agents in our model are LA agents, DG agents, VPP agents, MGCC agents and the DNO agent. These agents react by collecting inputs about their state and environment before any decision making (Wehinger, 2010).

Within a microgrid, the MGCC agent interacts with the VPP agents. The VPPs may be responsible for the management of LAs, DGs and ESSs. When a VPP agent is responsible for the management of other players, their communications are independently dealt from the rest of the simulation. This prevents the overflow of information through the system, since these particular communications are only important for the internal process between the VPP and its members. In fact, a VPP aggregation can be approached as a MAS by its own, with high interconnectivity with the global MAS system, particularly with the MGCC of the control area. The main responsibility and duty of the MGCC is to operate the microgrid through the interaction with all the participating agents in the microgrid and the upstream grid. It also controls and monitors all the microgrid protection devices (Kinoshita, 2010).

Agents make operational decisions locally with a high level of independence in comparison to a centralized system where they follow the instructions of microgrid managers (Logenthiran and Srinivasan, 2012). This approach is also more compatible with the structure of electricity markets. Implementing the financial incentive based DR programs by the VPPs influence the supply offers and demand bids that a MGCC is submitting to the market operator/DNO. The information is processed locally in the proposed energy management scheme. Accordingly, it saves the DNOs from facing the large data manipulation (Logenthiran, 2012). The proposed approach is integrated with MASGriP (Multi-Agent Smart Grid simulation Platform) (Oliveira et al., 2012), which is a system that has been developed for deep studies of the microgrid operation in an energy market environment (Dimeas and Hatziargyriou, 2005). This system allows analyzing the behavior of a high number of different intelligent agents acting in integrated microgrids.

The negotiation between the integrated microgrids reduces the cost of each microgrid through resource sharing at cheaper prices. Although the final solution may bring suboptimal solutions for VPPs with lower payoff compared with the dispatching in an isolated microgrid, the MGCC that is playing the role of the internal market operator can reduce the net operating cost of the microgrid. The ultimate objective of the proposed model is to reduce the operation cost of microgrids through negotiation with neighbour grids.

\section{CASE STUDY}

This case study demonstrates the advantages of the proposed approach. The three levels of negotiation are simulated, and the results are validated with real electricity markets data.

The microgrids of the test system (fig. 2) are assumed to have control, measurement and sensing devices with two-way communication structure where the smart operation is guaranteed with the dynamic responding of the agents. It is assumed that the whole system is operating at $25 \mathrm{kV}$. Part of the loads in each microgrid is fixed and part of them is responsive for financial incentives that can be in form of discounts on the regulated rates.

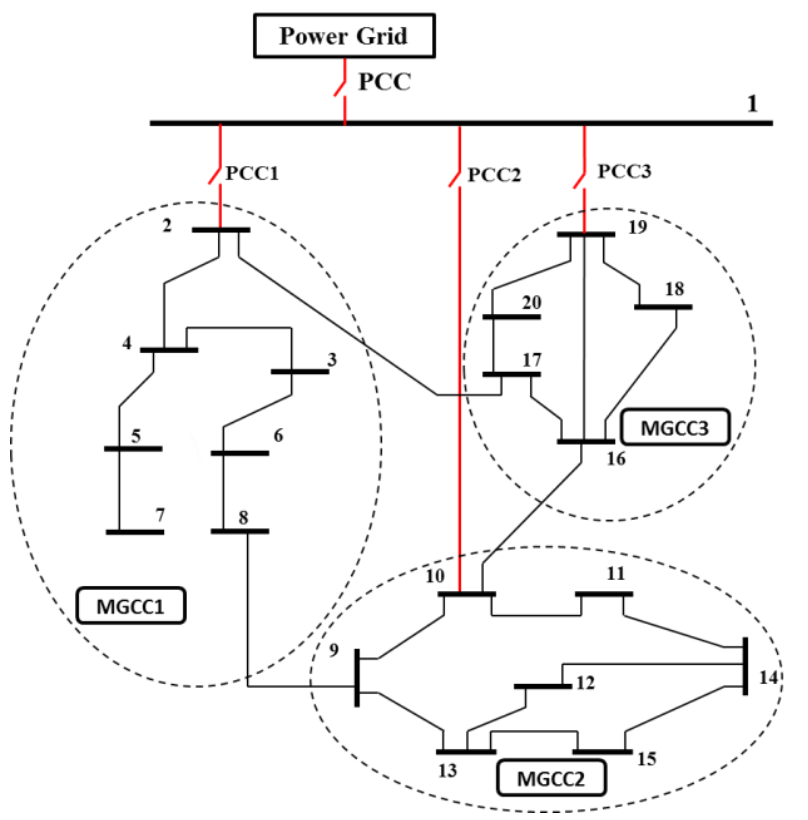

Fig. 2. Integrated microgrids.

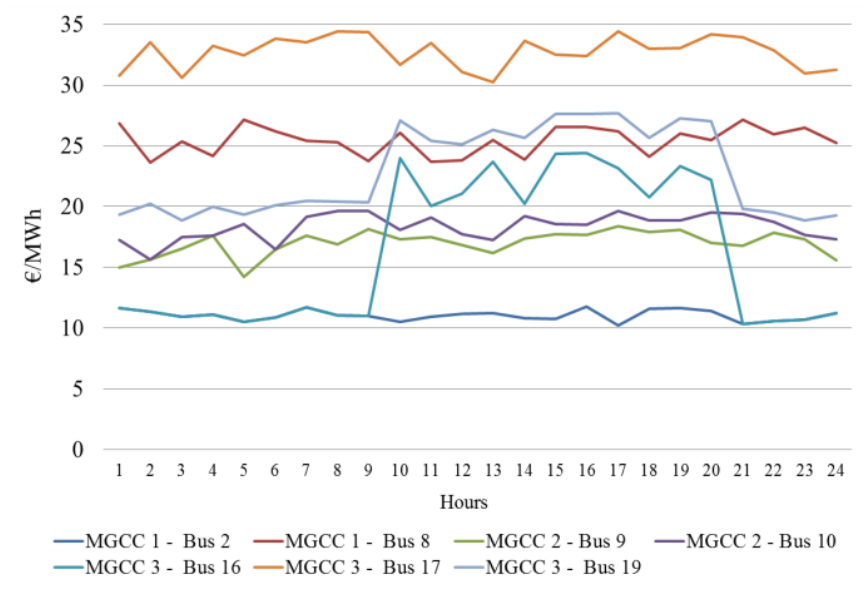

Fig. 3. Hourly LMPs at connection buses (internal market clearing). 
In the internal market clearing, MGCCs have expectations for the supply offers and/or demand bids from the upstream network and the interconnected microgrids at the joint buses. Based on these expectations and on the supply offers and demand bids of the internal VPPs, the MGCC determines the LMPs at the joint buses. MASGriP is interfaced with GAMS, in order to make optimal decisions for the VPPs offers and bids and the internal market clearing before the negotiation between MGCCs. The determined LMP values in the connection buses of the three considered microgrids, resulting from the internal microgrid market are presented in fig. 3 .

From fig. 3 it is visible that the LMPs of the connection buses of the three microgrids present rather variable values. These prices are used as reference values for the second and third steps of negotiation. The negotiation among the neighbor MGCCs considers these LMPs as the minimum accepting selling price or the maximum desirable buying price for power from the corresponding neighbor microgrids.

Considering the internal dispatch of each independent microgrid, we reach a situation where each of the microgrids, after supplying all the possible loads, is faced with one of two situations in each hour: having extra generation power, which is able to be sold; or a lack of supply, which must be bought:

- MGCC 1: Has power to sell in hours 1 to 7, 23 and 24;

- MGCC 2: Has extra generation in all hours of the day;

- MGCC 3: Sells from hours 1 to 7, and needs to buys on the remaining hours of the simulated day.

The three MGCCs negotiate with each other, trying to reach advantageous arrangements. For that, each of the MGCCs uses a negotiation strategy (section IV B): MGCC 1 assumes an Anxious position; MGCC 2 a Gluttonous approach; and MGCC 3 uses a Moderated personality. Figures 4 and 5 present the amounts of power that were traded between the three MGCCs, and a comparison between the established prices and the reference connection LMPs.

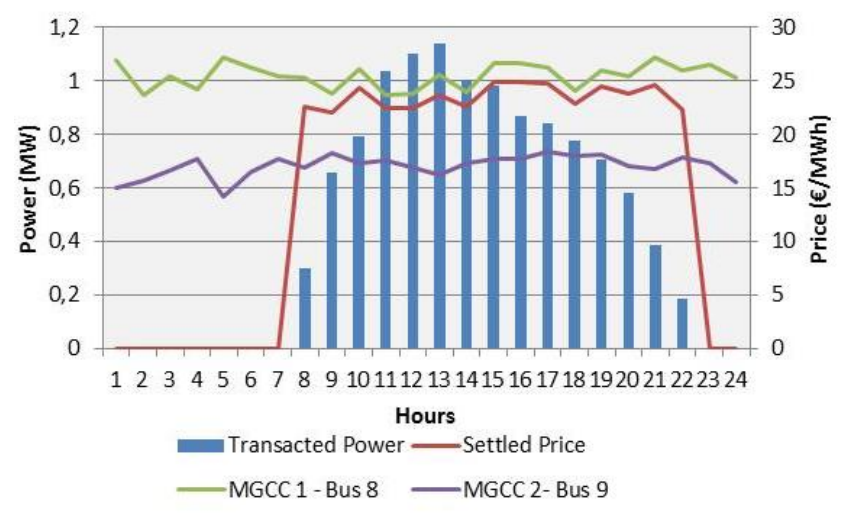

Fig. 4. Traded power between MGCC 1 and MGCC 2.

From fig. 4 one can see that MGCC 1 was able to buy all of its requiring power from MGCC 2, in all hours in which it presented a lack of supply. It is also visible that the achieved price is always located between the LMPs of the connection points between MGCC1 and MGCC 2 . This means that both MGCCs achieved favorable deals, since MGCC 1 was able to buy at prices below the LMP of the connection bus with MGCC 2, and MGCC 2 was able to sell at higher prices than the LMP in the connection bus with MGCC 1. Also, it is visible that the settled price is closer to the price of MGCC 1 than the one of MGCC 2. This is because of their negotiating natures.

The Anxious approach of MGCC 1 meant that it changed its demanded price very quickly, in the perspective of achieving a deal rapidly, which resulted in a fast disregard from the negotiation potential. MGCC 2's Gluttonous approach made this agent intransigent in the quest for the best possible deal, even if it meant not closing the deal at all.

From fig. 5 one can see that from hour 10 to hour 20 MGCC 3 bought power from MGCC 2. These are the hours in which the LMP of the connection bus of MGCC 3 with MGCC 2 were higher, and the only ones in which buying power from MGCC 2 would be favourable. Regarding the strategic behaviour, once again MGCC 2 was able to impose its Gluttonous personality, achieving nearly the best price.

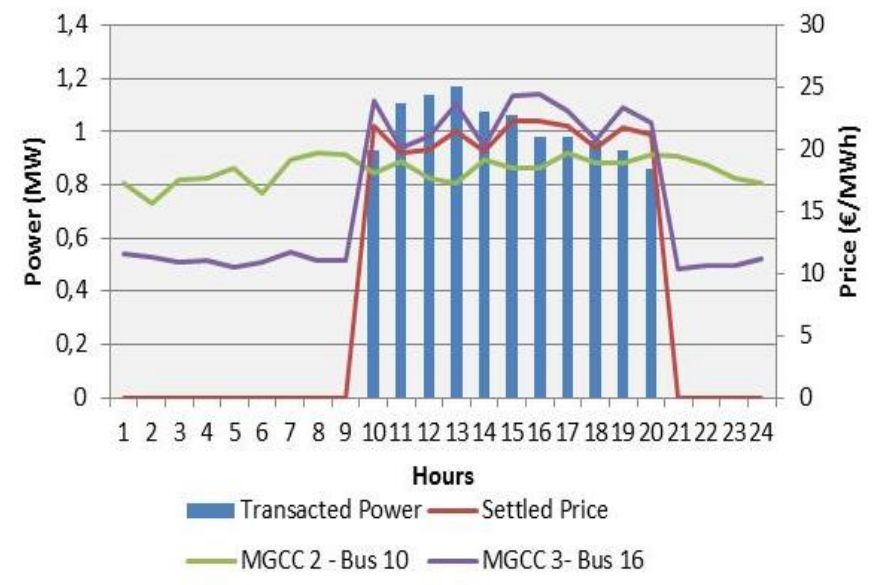

Fig. 5. Transactions between MGCC 1 and MGCC 3.

Regarding the third, and final level, the external electricity market, the generation of all microgrids is turned to its maximum, reaching for as much production as possible, in order to try to achieve the best revenues from the market. The market simulation is performed using MASCEM (Vale et al., 2011b), taking advantage on this simulator's ability to model the electricity markets using real data. For this simulation real players data from the Iberian market is used, making this simulation suitable for testing and validating the proposed approach in a realistic scenario, with the MGCCs competing with real electricity market players.

Fig. 6 shows the profits that each MGCC was able to achieve in the market. For the calculation of the profits, the real cost functions from all generators that are present in the three microgrids were considered. Fig. 6 shows that all three MGCC2 were able to achieve substantial profits from using the full generation of its generators as an opportunity to sell power in the market. 


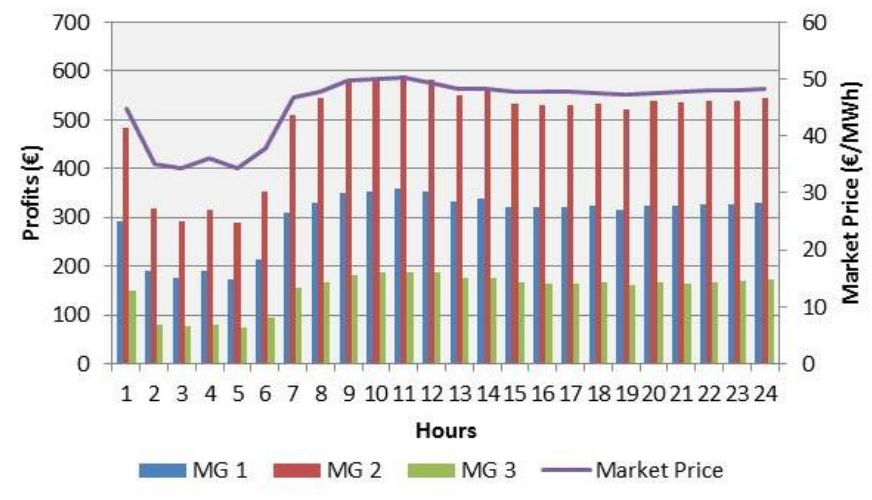

Fig. 6. Market results for the three considered MGCCs.

\section{CONCLUSIONS}

This paper studies the short-term operation of integrated microgrids in the market environment via MAS approach. The proposed model frees the DNOs from facing the difficulties of dealing with intermittent DGs and the load variations of integrated microgrids. This responsibility is put on the shoulders of local agents, MGCCs, VPPs and LAs. It saves the DNOs from the need for large data manipulations before the day-ahead markets, and gives players the opportunity to reach advantageous deals, depending on the negotiation opportunities that arise, and the contexts that are observed. The proposed MAS-based scheme simulates the interaction between decentralized and profit seeking entities, which the centralized approaches cannot represent.

The results show that the three negotiation levels can be complementary, providing microgrid operators with the chance of looking for the better arrangements. While the internal market is capable of assuring technical requirements in a much easier way; the negotiations with neighbour operators provides a means for reaching advantageous deals in certain situations, without the need for entering the electricity market, and with the obvious extra advantage for the network of getting or sending power to a much nearer geographic point. Finally, the external electricity market, is always present as an opportunity for entering negotiations for power that could not be sold or bought in the previous levels, or even serve as a strategic opportunity for negotiation.

\section{ACKNOWLEDGEMENTS}

This work is supported by FEDER Funds through COMPETE program and by National Funds through FCT under the projects FCOMP-01-0124-FEDER: PEstOE/EEI/UI0760/2011, and PTDC/SEN-ENR/122174/2010.

\section{REFERENCES}

Bo, Z., Xuesong, Z. \& Jian, C. (2012). Integrated microgrid laboratory system. Power Systems, IEEE Transactions on Sower Systems, 27, 2175-2185.
Bollen, M., Zhong, J., Samuelsson, O. \& Bjornstedt, J. (2009). Performance indicators for microgrids during grid-connected and island operation. IEEE PowerTech 2009, Bucharest.

Conejo, A. J., Carrión, M. \& Morales, J. M. (2010). Decision making under uncertainty in electricity markets, New York, Springer.

Dimeas, A. L. \& Hatziargyriou, N. D. (2005). Operation of a multiagent system for microgrid control. IEEE Transactions on Power Systems, 20, 1447-1455.

Duy Thanh, N., Michael, N. \& Martin De, G. (2012). Walrasian market clearing for demand response exchange. IEEE Transactions on Power Systems, 27, 535-544.

Fotouhi Ghazvini, M. A., Morais, H. \& Vale, Z. (2012). Coordination between mid-term maintenance outage decisions and short-term security-constrained scheduling in smart distribution systems. Applied Energy, 96, 281291.

Kinoshita, T. (2010). A multiagent system for microgrid operation in the grid-interconnected mode. Journal of Electrical Engineering \& Technology, 5, 246-254.

Liu, H., Tesfatsion, L. \& Chowdhury, A. (2009). Locational marginal pricing basics for restructured wholesale power markets. IEEE Power and Energy Society General Meeting 2009.

Logenthiran, T. (2012). Multi-Agent System for Control and Management of Distributed Power Systems. Ph.D. thesis, National University of Singapore.

Logenthiran, T. \& Srinivasan, D. (2012). Multi-agent system for the operation of an integrated microgrid. Journal of Renewable and Sustainable Energy, 4, 013116.

Oliveira, P., Pinto, T., Morais, H. \& Vale, Z. (2012). MASGriP ; A Multi-Agent Smart Grid Simulation Platform. IEEE Power and Energy Society General Meeting 2012.

Rasheduzzaman, M., Bhaskar, S. N. \& Chowdhury, B. H. (2012). Implementation of a microgrid central controller in a laboratory microgrid network. North American Power Symposium (NAPS).

Salam, A., Mohamed, A. \& Hannan, M. (2008). Technical challenges on microgrids. ARPN Journal of Engineering and Applied Sciences, 3, 64-69.

Vale, Z., Pinto, T., Morais, H., Praca, I. \& Faria, P. (2011a). VPP's multi-level negotiation in smart grids and competitive electricity markets. IEEE Power and Energy Society General Meeting 2011.

Vale, Z., Pinto, T., Praca, I. \& Morais, H. (2011b). MASCEM: Electricity markets simulation with strategic agents. intelligent systems, IEEE Intelligent Systems, 26, 9-17.

Wehinger, L. A. (2010). Agent-based modeling in electricity markets: Introducing a new predictive agent learning approach. Master's thesis, Swiss Federal Institute of Technology Zurich, Zurich, Switzerland.

Wooldridge, M. (2008). An introduction to multiagent systems, Wiley. 